\title{
Faster Healing Process by Sparing Intramural Myoma's Pseudocapsule during Laparoscopic Myomectomy Compared with Removing It during Open Myomectomy
}

\author{
Athanasios Zikopoulos1, Yannis Prapas², Maria Paraskevaidi ${ }^{3}$, Charalampos Siristatidis ${ }^{4}$, \\ Apostolia Galani ${ }^{5}$, Orestis Tsonis ${ }^{6}$, Minas Paschopoulos ${ }^{6}$, Konstantinos Zikopoulos ${ }^{6}$, \\ Efstratios Kolibianakis ${ }^{7}$ \\ ${ }^{1}$ Department of Obstetrics and Gynaecology, Royal Cornwall Hospital, Cornwall, UK \\ ${ }^{2}$ IAKENTRO, Infertility Treatment Centre, Thessaloniki, Greece \\ ${ }^{3}$ Institute of Reproductive and Developmental Biology, Department of Metabolism, Digestion and Reproduction, Faculty of \\ Medicine, Imperial College London, London, UK \\ ${ }^{4}$ Second Department of Obstetrics and Gynaecology/Assisted Reproduction Unit, Faculty of Medicine, Aretaieion Hospital, \\ National and Kapodistrian University of Athens, Athens, Greece \\ ${ }^{5}$ Department of Obstetrics and Gynaecology, Royal Preston Hospital, Preston, UK \\ ${ }^{6}$ Department of Obstetrics and Gynaecology/Assisted Reproduction, Faculty of Medicine, University of Ioannina, \\ Ioannina, Greece \\ ${ }^{7}$ Unit for Human Reproduction, 1st Department of Obstetrics and Gynaecology, Medical School, Aristotle University of \\ Thessaloniki, Thessaloniki, Greece \\ Email: thanzik92@gmail.com
}

How to cite this paper: Zikopoulos, A., Prapas, Y., Paraskevaidi, M., Siristatidis, C., Galani, A., Tsonis, O., Paschopoulos, M., Zikopoulos, K. and Kolibianakis, E. (2021) Faster Healing Process by Sparing Intramural Myoma's Pseudocapsule during Laparoscopic Myomectomy Compared with Removing It during Open Myomectomy. International Journal of Clinical Medicine, 12, 424-432.

https://doi.org/10.4236/ijcm.2021.1210038

Received: August 4, 2021

Accepted: October 11, 2021

Published: October 14, 2021

\begin{abstract}
Uterine fibroids are the most common benign tumours in the reproductive system. They are proliferations of smooth muscle cells of the myometrium containing a large quantity of extracellular matrix and they are surrounded by a pseudo capsule of compressed areolar tissue and smooth muscle cells. They can cause various symptoms such as menorrhage, pain and infertility and therefore they can be a traumatic experience for several women. The treatment of choice is myomectomy. In the past, myomectomy was performed by relatively atraumatic techniques, which involved stretching the myoma from its pseudocapsule to extract the fibroid directly from the surrounding fibromuscular tissue, breaking up the fibrous bridge. Modern laparoscopic intracapsular myomectomy (LIM), however, leaves the fibrovascular network surrounding the myoma (namely the "fibroid neurovascular bundle") intact which reduces the bleeding and/or uterine musculature trauma, and spares the neuropeptide fibers of the pseudocapsule. In this observational study, we com-
\end{abstract}


Copyright $\odot 2021$ by author(s) and Scientific Research Publishing Inc. This work is licensed under the Creative Commons Attribution International License (CC BY 4.0).

http://creativecommons.org/licenses/by/4.0/ pare the two techniques-laparoscopic intracapsular myomectomy (LIM) and conventional abdominal myomectomy (CAM) regarding the longterm uterine healing and a significantly faster healing process of the uterine incision was achieved by LIM compared to CAM.

\section{Keywords}

Myomectomy, Laparoscopic Intracapsular Myomectomy, Healing

\section{Introduction}

Uterine leiomyomas, (also known as fibroids or myomas), are the most common benign tumours in the reproductive system of childbearing age women, with a prevalence of $30 \%-35 \%$ [1] [2] [3]. However, fibroids typically regress during menopause since they are oestrogen dependent.

Uterine leiomyomas are proliferations of smooth muscle cells of the myometrium containing a large quantity of extracellular matrix (fibronectin, collagen, proteoglycan), and are surrounded by a pseudocapsule of compressed areolar tissue and smooth muscle cells. This pseudocapsule contains very few blood vessels and lymphatic vessels.

Myomectomy is the treatment of choice for symptomatic women who have symptoms and who wish to preserve their future fertility. The overall risk of uterine rupture during pregnancy or labour after myomectomy is $2.4 / 1000$ between 29 and 35.5 weeks and is attributed to the extensive muscular damage or excessive use of diathermocoagulation [3]. To minimize the risk, preservation of the uterine anatomical and functional integrity during myomectomy, especially in women of reproductive age, is of imminent importance. The restoration and maintenance of the physiologic function of the uterus is or should be, the ultimate goal of surgical treatment.

In the past, myomectomy was performed by relatively atraumatic techniques, which involved stretching the myoma from its pseudocapsule to extract the fibroid directly from the surrounding fibromuscular tissue, breaking up the fibrous bridge. Modern laparoscopic intracapsular myomectomy (LIM), however, leaves the fibrovascular network surrounding the myoma (namely the "fibroid neurovascular bundle") intact which reduces the bleeding and/or uterine musculature trauma, and spares the neuropeptide fibers of the pseudocapsule. This more recent approach has a favourable impact on the uterine healing and successive functionality, has simplified the surgical procedure and minimised the concerns over blood loss, post-surgical adhesion and wound healing [4] [5]. Using the LIM techniques, the risk of uterine rupture during future pregnancy or labour, appears to be decreased, although the issue is still under debate.

The quality of healing of the myometrial incision after myomectomy can be evaluated by ultrasound [6] [7]. 
The objective of the present prospective observational study was to compare conventional abdominal myomectomy with LIM in premenopausal women in order to assess the quality of uterine healing.

\section{Materials and Methods}

A prospective observational study was performed from January 2017 to December 2020 in order to compare laparoscopy intracapsular myomectomy (LIM) with conventional abdominal myomectomy (CAM) with removal of the pseudocapsule in premenopausal women. LIM was performed at IAKENTRO IVF Center and CAM was performed at the University Hospital of Ioannina.

This study was approved by the local Ethics Committee of both institutions. Informed consent was obtained from all participants prior to their inclusion in the study.

\subsection{Inclusion and Exclusion Criteria}

Study participants were women of reproductive age with an indication of myomectomy who required preservation of their future fertility. All of the participants were of caucasian origin. The myomas were intramural or sub-serous (FIGO types 3 - 6) of 4 - $14 \mathrm{~cm}$ diameter. The exclusion criteria included: no other gynaecological diseases detected on preoperative ultrasound, no previous history of pelvic or abdominal surgery or pelvic inflammation, suspicion of genital cancer and/or uncertain pathology of genital tract, presence of, adnexal masses, endometrial hyperplasia with or without atypia. Suspected adenomyosis and/or adenomyosis were also excluded from the study (Table 1). The patients' demographics, such as mean age (standard deviation [SD]), parity, body mass index (BMI; $\mathrm{kg} / \mathrm{m}^{2}$ ), were recorded (Table 2).

\subsection{Study Protocol}

Preoperatively all patients were evaluated preoperatively by transvaginal ultrasound within 30 days before surgery in which the number of myomas, size and location were recorded. A pelvic magnetic resonance imaging (MRI) with contrast

Table 1. Inclusion and exclusion criteria.

\begin{tabular}{ll}
\hline \multicolumn{1}{c}{ Inclusion Criteria } & \multicolumn{1}{c}{ Exclusion criteria } \\
\hline 1) Reproductive age & $\begin{array}{l}\text { 1) No other gynaecological diseases detected on } \\
\text { preoperative ultrasound }\end{array}$ \\
$\begin{array}{ll}\text { 2) Caucasian Origin } & \text { 2) No previous history of pelvic or abdominal } \\
\text { surgery or pelvic inflammation or suspicion of } \\
\text { genital cancer }\end{array}$ \\
$\begin{array}{ll}\text { 3) Fibroids (intramural or sub-serosal confirmed } \\
\text { with investigation (pelvic scan, MRI, CT) }\end{array}$ & $\begin{array}{l}\text { 3) presence of adnexal masses, endometrial } \\
\text { hyperplasia with or without atypia. }\end{array}$ \\
4) No suspected adenomyosis & \\
\hline
\end{tabular}


Table 2. Epidemiologic characteristics of patients included in the study. LIM: laparoscopy intracapsular myomectomy; CAM: conventional abdominal myomectomy.

\begin{tabular}{cccc}
\hline Characteristics & Overall $(\mathrm{n}=106)$ & LIM $(\mathrm{n}=75)$ & CAM $(\mathrm{n}=31)$ \\
\hline Age $($ years $)$ & 32.5 & 34 & 31 \\
BMI $\left(\mathrm{kg} / \mathrm{m}^{2}\right)$ & 27 & 25 & 29 \\
Nulliparous & 88 & 62 & 26 \\
Infertility & 93 & 65 & 28 \\
Number of Fibroids & 2.6 & 2.2 & 3.1 \\
Previous Abdominal surgery & 31 & 23 & 8 \\
Diameter of fibroids $(\mathrm{mm})$ & 77.5 & 75 & 80 \\
\hline
\end{tabular}

medium also was also performed the same period in order to confirm the findings of the transvaginal ultrasound and detect a potential leiomyosarcoma. At the end of the surgery the extracted myomas were sent for histological evaluation.

Myomectomies were performed between day 7 and day 17 of the menstrual cycle. During this interval, oestrogen proliferative activity has not yet maximized endometrial thickness, while uterine vascularization is more limited compared to the second luteal phase of menstrual cycle. No preoperative treatment with $\mathrm{GnRH}$ analogues or Ulipristal Acetate was used in either group.

In order to evaluate and compare the healing process, all patients were monitored postoperatively by ultrasound on days 7, 30 and 90 after the operation (Table 3). All women were advised to avoid any pregnancy for the first three months postoperatively and were all asked subsequently to set report potential adverse outcomes or conception.

The surgical protocol and LIM technique have been described in detail in previous studies [6]. Evaluation of wound healing assessed progressive decrease of uterine incision size (initial, final and \% difference), time period of healing and the existence of anechoic areas, hematomata.

As wounds fail to progress through normal stage of healing, they become more differential regarding shape, size and the extent of tissue damage, which is compounded by the absence of uniformly acceptable wound healing diagnostic methods.

\section{Statistics}

Continuous variables were expressed as mean $\pm \mathrm{SD}$, while categorical variables as n (\%). Kolmogorov-Smirnov test was used to assess normality of values distribution for continuous variables. Chi-square test was used to compare categorical variables, while continuous variables were compared with Independent samples t-test in case of normally distributed values or Mann-Whitney test in case of abnormally distributed values. Statistical analysis was performed with Statistical Package for Social Science 21.0. 
Table 3. Final outcomes comparing the two techniques.

\begin{tabular}{ccc}
\hline Surgical Outcomes & LIM $(\mathrm{n}=75)$ & CAM $(\mathrm{n}=31)$ \\
\hline Primary Outcomes & $2 \pm 0.6$ & $3 \pm 0.8$ \\
Uterine incision (maximum, cm) & $28 \pm 8.2$ & $37 \pm 9$ \\
Time period to healing (days, mean \pm SD) & $65.4 \pm 24.3$ & $87 \pm 34$ \\
Operative time (min; mean \& SD) & 0 & 0 \\
Urologic injury & 0 & 0 \\
Intestinal Injury & 0 & $2.8 \pm 0.5$ \\
Vascular Injury & $1.6 \pm 0.8$ & \\
\hline
\end{tabular}

\section{Results}

Overall, 106 women were included in the study, out of which 75 in the LIM group (group 1I) and 31 in the CAM group (group II).

Mean number of extracted myomas was 2.2 for group I and 3.1, for group II, while mean diameter of the largest myoma was $75 \mathrm{~mm}$ in group I and $80 \mathrm{~mm}$ in group II. Epidemiological characteristics of patients are presented in Table 1.

On the $7^{\text {th }}$ postoperative day, the site of the LM or AM scar appeared as a highly echogenic area with profuse blood flow at the periphery in all cases. Four patients (4\%) had a hematoma, in group II, which appeared as a hypoechoic area with areas of echogenicity. The anechoic-hypoechoic area was almost avascular (volume $15 \mathrm{~cm}^{3}, 21 \mathrm{~cm}^{3}, 91 \mathrm{~cm}^{3}$ and $95 \mathrm{~cm}^{3}$ ).

By the $9^{\text {th }}$ postoperative month, the LM or AM healing area had regained the echogenicity of normal myometrium, however a hematoma appearing as a hypoechoic area, with decreased volume from $95 \mathrm{~cm}^{3}$ to $9 \mathrm{~cm}^{3}$ and from $91 \mathrm{~cm}^{3}$ to $5 \mathrm{~cm}^{3} 3$ was still present in 2 cases. The non-operated myometrial areas showed significantly more prominent blood flow than the healing areas.

The time period until healing was significantly increased in CAM $(P=0.001)$. Wound sign disappeared in $90 \%$ of the patients 30 days postoperatively in group I but in only $30 \%$ in group II. However, ultrasonographic wound signs disappeared in all study patients on the $90^{\text {th }}$ postoperative day. Estimated blood loss and, operative time were significantly lower in cases treated with LIM. This is obvious as the dissection of myoma is easier leaving the pseudocapsule and the bed of the myomectomy is usually free from haemorrhage.

\section{Discussion}

This prospective observational study showed a significantly faster healing process of the uterine incision with LIM to sparing the psedocapsule compared to CAM.

This finding reflects the clinical observation that sparing the pseudocapsule reduces surgical trauma, leaves less myometrial defects, uses less or no electrocautery and therefore enables a faster healing process.

Generally, an optimal myomectomy aims to preserve the integrity of the extra- 
cellular matrix, either performed via laparoscopy or laparotomy, as this ensures the three-dimensional organization of the myometrium after myomectomy [8] [9]. This is especially important in women of reproductive age who have not yet completed their family.

In recent years, laparoscopic myomectomy has been considered as an alternative of laparotomy with numerous advantages including shorter hospitalisation, less need for analgesia, lower intraoperative blood loss and a favourable outcome in a subsequent pregnancy. Although uterine rupture during pregnancy, labour or delivery may still occur, the risk is low.

The wound healing process begins with the inflammatory phase lasting up to $72 \mathrm{~h}$ during which pain, swelling, redness and increased local temperature are common symptoms. Accumulation of exudates and edema begins the process of tissue repair following injury when a blood clot forms and seals the area. In muscular injuries, a myofilament reaction takes place and peripheral muscle fiber contraction occurs within the first $2 \mathrm{~h}$. Edema and anoxia results in cell damage and death within the first $24 \mathrm{~h}$, and the release of protein breakdown products from damaged cells leads to further edema, tissue hypoxia and cell death. Phagocytosis then begins to rid the area of cell debris and edema [10]. The muscular regeneration and repair occur during the fibro-elastic/collagenforming phase, which lasts from $48 \mathrm{~h}$ up to 6 weeks. During this time, structures are rebuilt, and regeneration occurs. Fibroblast cells begin to produce Type III collagen at 4 days, and fiber organization is random and immature. Capillary budding occurs, bringing nutrients to the region, and collagen cross-linking begins. As the process proceeds, the number of fibroblasts decreases as more collagen is laid down. This phase ends with the beginning of wound contracture and shortening of the margins in the injured area. The last phase of healing is the remodeling phase, which lasts from 3 weeks to 12 months. Gradually, crosslinking and shortening of the collagen fibers promote the formation of a tight, strong scar. Final aggregation, orientation and arrangement of collagen fibers occur during this phase. Healing of the injured muscle does not fully restore muscle to its prior state, as fibrous scar tissue slows muscle healing. The two processes of healing and fibrosis compete and impair complete regeneration [11]. Many of these processes are catalysed by growth factors such as transforming growth factor-beta 1 (TGF-b1), a ubiquitous substance that initiates a cascade of events that activate both myogenesis and fibrosis, and decorin, a proteoglycan that impedes fibrosis by binding to TGF-b1 [12]. These growth factors are under active investigation to determine their role in muscle healing.

Myomectomy always causes trauma due to the visceral peritoneal incision, opening of the myometrium, removal of the fibroid and closure of the muscle margins by suture. Each major structural and vascular disruption during myomectomy leads to a classical inflammatory reaction and causes irreversible tissue damage and cell necrosis with two plausible options. A regenerative response results in an optimal reconstruction of the damaged tissue and a high-quality 
muscle scar. A fibrotic response results in a poor reconstruction of the myometrium and a fibrotic and inelastic scar. In the first case, the myometrium returns to full function, while in the second case the result is tissue dysfunction characterised by pain, inflammation and altered internal tissue stress. The injury may result in progressive functional disability and a higher risk of uterine rupture during pregnancy or during labour. The progressive evolution of the uterine scar from a highly echogenic area with an ill-defined heterogeneous myometrial texture to a normal echoic area confirms a normal healing process, whereas a fibrotic scar continues to display hyper or hypoechogenic areas and increased mean scar diameter.

When surgeon gently removes the UF through the pseudocapsule with selective coagulation of its vessel, they preserve the muscle surrounding UF and avoids excessive bleeding, returning it to normal healthy uterine tissue. Extensive diathermocoagulation and excessive i.m. bleeding generally worsens the healing process and myometrial function in subsequent pregnancy [12].

Moreover, some of the pseudocapsule vessels join at the base of the UF creating a little foot that often bleeds during an extra-capsular myomectomy, which creates a hematoma, manifestating as avascular echoic area.

The myometrial area of the scar after myomectomy can be evaluated by $2 \mathrm{D}$ ultrasound and Doppler velocimetry, a non-invasive and, safe method that assesses uterine perfusion, the presence of post-operative hematoma and disechogenic, heterogeneous, or an ill-defined scar area, all unfavourable signs for myometrial scarring.

\section{Conclusions}

Intracapsular myomectomy enhances myometrium integrity peripheral to the fibroid site, by preserving the neurovascular bundle and neurotransmitters surrounding fibroids, for uterine healing and myometrium restoration after surgery. Moreover, allowing correct myometrial healing, intracapsular myomectomy could preserve reproductive outcomes and normal labour and delivery and permit less bleeding, better neurovascular bundle sparing for scar quality and reductions in post-operative adhesions. Increasing knowledge of the pseudocapsule angiogenesis and neurovascular fibers could play an important role in understanding the origin and recurrence of uterine leiomyomas, therefore allowing for optimal treatments.

In the present study, we demonstrated that dissection of the fibroid should run inside the avascular plane, leaving the pseudocapsule around the outside. The bed of the myomectomy is usually free from haemorrhage at the end of dissection if care has been taken to follow the avascular cleavage plane; there is no need to take further steps for haemostasis. Therefore, independent of the way of surgery laparoscopy or abdominal this study has demonstrated that there is no need to. Remove the pseudocapsule during myomectomy, which is independent of the use of a laparoscopic or abdominal surgical procedure. 
In conclusion, the current study has shown that a faster healing process is expected after laparoscopic myomectomy when the intamural myoma's pseudocapsule is spared compared with its removal during open myomectomy.

\section{Conflicts of Interest}

The authors declare no conflicts of interest regarding the publication of this paper.

\section{References}

[1] Tinelli, A., Malvasi, A., Hurst, B.S., Tsin, D.A., Davila, F., Dominguez, G., Dell'edera, D., Cavallotti, C., Negro, R., Gustapane, S., Teigland, C.M. and Metaller, L. (2012) Surgical Management of Neurovascular Bandle in Uterine Fibroid Pseudocapsule. JSLS, 16, 119-129. https://doi.org/10.4293/108680812X13291597716302

[2] Tinelli, A., Hurst, B.S., Hudelist, G., Tsin, D.A., Stark, M., Mettler, L., Guido, M. and Malvasi, A. (2012) Laparoscopic Myomectomy Focusing on the Myopia Pseudocapsule: Technical and Outcome Reports. Human Reproduction, 27, 427-435. https://doi.org/10.1093/humrep/der369

[3] Bonney, V. (1931) The Technique and Results of Myomectomy. Lancet, 217, 171-177. https://doi.org/10.1016/S0140-6736(00)40479-4

[4] Malvasi, A., Tinelli, A., Cavallotti, C., Morroni, M., Tsin, D.A., Nezhat, C., Stark, M. and Mettler, L. (2011) Distribution of Substance P (SP) and Vasoactive Intestinal Peptide (VIP) in Pseudocapsules of Uterine Fibroids. Peptides, 32, 327-332. https://doi.org/10.1016/j.peptides.2010.10.034

[5] Beyth, Y., Jaffe, R. and Goldberqer, S. (1992) Uterine Remodelling Following Conservative Myomectomy: Ultrasonographic Evaluation. Acta Obstetricia et Gynecologica Scandinavica, 71, 632-635. https://doi.org/10.3109/00016349209006233

[6] Tinelli, A., Hurst, B.S., Mettler, L., Tsin, D.A., Pellegrino, M., Nicolardi, G., Dell'Edera, D. and Malvasi, A. (2012) Ultrasound Evaluation of Uterine Healing after Laparoscopic Intracapsular Myomectomy: An Observational Study. Human Reproduction, 27, 2664-2670. https://doi.org/10.1093/humrep/des212

[7] Prapas, Y., Zikopoulos, A., Petousis, S., Xiromeritis, P., Tinelli, A., Ravanos, K., Margioula-Siarkou, C., Chalkia-Prapa, E.-M. and Prapas, N. (2020) Single Layer Suturing in Intracapsular Myomectomy of Intramural Myomas Is Sufficient for a Normal Wound Healing. European Journal of Obstetrics \& Gynecology and Reproductive Biology, 248, 204-210. https://doi.org/10.1016/j.ejogrb.2020.03.042

[8] Frishman, G.N. and Jurema, M.W. (2005) Myomas and Myomectomy. Journal of Minimally Invasive Gynecology, 12, 443-448. https://doi.org/10.1016/j.jmig.2005.05.023

[9] Huard, J., Li, Y. and Fu, F.H. (2002) Muscle Injuries and Repair: Current Trends in Research. Journal of Bone and Joint Surgery-American Volume, 84, 822-832. https://doi.org/10.2106/00004623-200205000-00022

[10] Tidball, J.G. and Villalta, S.A. (2010) Regulatory Interactions between Muscle and the Immune System during Muscle Regeneration. American Journal of PhysiologyRegulatory, Integrative and Comparative Physiology, 298, R1173-R1187. https://doi.org/10.1152/ajpregu.00735.2009

[11] Li, Y., Foster, W., Deasy, B.M., Chan, Y., Prisk, V., Tang, Y., Cummins, J. and Huard, J. (2004) Transforming Growth Factor-Betal Induces the Differentiation of Myo- 
genic Cells into Fibrotic Cells in Injured Skeletal Muscle: A Key Event in Muscle Fibrogenesis. The American Journal of Pathology, 164, 1007-1019.

https://doi.org/10.1016/S0002-9440(10)63188-4

[12] Mettler, L., Tinelli, A., Hurst, B.S., Teigland, C.M., Sammur, W., Dell'edera, D., Negro, R., Gustapane, S. and Malvasi, A. (2011) Neurovascular Bundle in Fibroid Pseudocapsule and Its Neuroendocrinologic Implications. Expert Review of Endocrinology \& Metabolism, 6, 715-722. https://doi.org/10.1586/eem.11.62 